TRANSACTIONS OF THE

AMERICAN MATHEMATICAL SOCIETY

Volume 352, Number 5, Pages 2319-2330

S 0002-9947(99)02576-3

Article electronically published on November 17, 1999

\title{
A CONSTRUCTION OF HOMOLOGICALLY AREA MINIMIZING HYPERSURFACES WITH HIGHER DIMENSIONAL SINGULAR SETS
}

\author{
NATHAN SMALE
}

\begin{abstract}
We show that a large variety of singular sets can occur for homologically area minimizing codimension one surfaces in a Riemannian manifold. In particular, as a result of Theorem $\mathrm{A}$, if $N$ is smooth, compact $n+1$ dimensional manifold, $n \geq 7$, and if $S$ is an embedded, orientable submanifold of dimension $n$, then we construct metrics on $N$ such that the homologically area minimizing hypersurface $M$, homologous to $S$, has a singular set equal to a prescribed number of spheres and tori of codimension less than $n-7$. Near each component $\Sigma$ of the singular set, $M$ is isometric to a product $C \times \Sigma$, where $C$ is any prescribed, strictly stable, strictly minimizing cone. In Theorem B, other singular examples are constructed.
\end{abstract}

\section{INTRODUCTION}

In 1960, Federer and Flemming [6] developed the theory of rectifiable currents, with which they were able to address some fundamental minimization problems in geometry. The one we are interested in here, is the following. Given a smooth, compact Riemannian manifold $(N, g)$, of dimension $n+1$, and given a non-zero, integer homology class $\gamma \in H_{k}(N, \mathbf{Z}), 1 \leq k \leq n$, does there exist a $k$-dimensional surface $M \subset N$ (possibly with multiplicity), representing $\gamma$, which has the smallest area among all such surfaces? This was answered affirmatively in [6] by allowing $M$ to be in a generalized class of surfaces called integral currents, which the authors defined. A priori, an integral current is supported in a $k$-dimensional rectifiable set, and thus it can have very general singularities. Indeed, if $k<n$ in the above problem, there may be topological obstructions to the existence of a smooth submanifold representing $\gamma$, although for the hypersurface case $(k=n)$, there are no such obstructions. For codimension one, homologically area minimizing currents, there is a partial regularity theory, [5], [11], and [4], which says that the singular set of such currents has Hausdorff dimension at most $n-7$, if $n \geq 7$ (consisting of isolated points if $n=7$ ), and is empty when $n<7$. Such results also hold in the context of area minimizing currents with a given boundary.

The first example of an area minimizing hypersurface with boundary, having a singularity, was given by Bombieri, De Giorgi, and Giusti [2], where they showed that the truncated cone over $S^{p} \times S^{p}$ in $\mathbf{R}^{2 p+2}$ with $p \geq 3$, was area minimizing. Further examples were given by Hardt and Simon [8], where they showed that certain perturbations of truncated cones constructed earlier by Caffarelli, Hardt and

Received by the editors January 30, 1998.

2000 Mathematics Subject Classification. Primary 53A10; Secondary 49Q05. 
Simon [3], were area minimizing. In a previous paper [12, the author constructed the first examples of homologically area minimizing hypersurfaces in Riemannian manifolds, having singularities. There, it was shown that some smooth manifolds (in particular $S^{n} \times S^{1}$ with $n \geq 7$ ) admitted metrics such that the area minimizing currents in certain codimension one homology classes had two isolated singularities. Near the singularities, the minimizer looked like any prescribed strictly stable, strictly minimizing, regular hypercone (see 8 for a discussion of these cones). Here, using the same techniques, together with some new ideas, we show how to construct examples with higher dimensional singular sets. We can also, essentially prescribe the homology class. In particular we prove the following theorem.

Theorem A. Let $N$ be a smooth, compact Riemannian manifold of dimension $n+1 \geq 8$, and let $S$ be a smooth, connected, oriented, embedded hypersurface which represents a nontrivial element of $H_{n}(N, \mathbf{Z})$. Let $\Sigma_{1}, \ldots, \Sigma_{L}$ be any collection of smooth manifolds, such that $\operatorname{dim} \Sigma_{i}=k_{i} \leq n-7$, and each $\Sigma_{i}$ is either a standard $k_{i}$-sphere (or a point if $k_{i}=0$ ), or a $k_{i}$ dimensional torus. Let $C_{1}, \ldots, C_{L}$ be a collection of strictly stable, strictly minimizing, regular hypercones, with $\operatorname{dim}_{i}+$ $k_{i}=n$. Then there is a metric $g$ on $N$, such that the area minimizing current homologous to $S$, consists of a multiplicity one, connected hypersurface $M$, whose singular set is a union of submanifolds $\bigcup_{i} \Lambda_{i}$, where $\Lambda_{i}$ is diffeomorphic to $\Sigma_{i}$. Near each $\Lambda_{i}$, $M$ looks like a product $C_{i} \times \Sigma_{i}$.

Actually, a somewhat stronger and more precise result is proven; the $\Sigma_{i}$ can belong to a larger class of manifolds (see Theorem 1, of section 2). We also prove another result, which states that essentially any union of manifolds of appropriate codimension can occur as the singular set of some homologically minimizing current in some Riemannian manifold, although here we cannot prescribe the homology class, and the ambient manifold depends on the singular set one wishes to prescribe.

Theorem B. Let $n \geq 7$, and let $\Sigma_{1}, \ldots, \Sigma_{L}$, be any collection of smooth, compact, oriented manifolds with $\operatorname{dim} \Sigma_{i}=k_{i} \leq n-7$. Let $C_{1}, \ldots, C_{L}$ be any collection of strictly stable, strictly minimizing, regular hypercones with $\operatorname{dim} C_{i}+\operatorname{dim} \Sigma_{i}=$ $n$. Then there is a smooth, compact, orientable Riemannian manifold $(N, g)$ of dimension $n+1$, which supports a homologically area minimizing current, which consists of a connected, multiplicity one hypersurface $M$, with singular set equal to a union $\bigcup_{i} \Lambda_{i}$, where each $\Lambda_{i}$ is diffeomorphic to $\Sigma_{i}$. Near $\Lambda_{i}$, M looks like a product $C_{i} \times \Sigma_{i}$.

See Theorem 2 in section 2, for a more precise result. The construction of $N$ is fairly explicit, and there is a large class of such manifolds. The more difficult aspects of the proofs of the above theorems already appeared in [12. In both theorems, the basic idea, is to first construct a hypersurface $M$, and a metric on $N$, so that $M$ has the desired properties near it's singular set. The metric is then perturbed so that $M$ is stationary. Then it is perturbed again so that $M$ becomes stable (this was already done in [12]). It is then shown that if $M$ is stable, and if the singular set has an appropriate product structure, then $M$ is actually homologically minimizing in some neighborhood. The metric is then sufficiently enlarged on a complement of such a neighborhood, such that $M$ becomes globally homologically minimizing.

I would like to thank the I.H.E.S for its' hospitality. This paper was written, while visiting there on sabattical. 


\section{SOME TECHNICAL LEMMAS}

In this section, $(N, g)$ will denote a smooth, compact, Riemannian manifold of dimension $n+1$, with $n \geq 7$. We will denote by $M$, a subset of $N$ with certain regularity properties. On the complement of a closed singular set, $\operatorname{sing}(M)$ of Hausdorff dimension $\leq n-7$, we will assume that $M / \operatorname{sing}(M)$ is a smooth, orientable, $n$-dimensional submanifold of $N$. Let $d(\cdot, \cdot)$ be the distance function in $N$ (relative to $g$ ), and for a closed set $K \subset N$ let $d(x, K)=\inf _{y \in K} d(x, y)$. For $\sigma>0$, and $\delta>0$ define $\mathcal{N}(\sigma)=\{x \in N: d(x, \operatorname{sing}(M))<\sigma\}$, and $\mathcal{U}(\delta)=\{x \in N: d(x, M)<\delta\}$. Recall that a regular, minimal hypercone $C$ in $\mathbf{R}^{m+1}$, is a minimal surface of the form

$$
C=\{r \theta: 0 \leq r<\infty, \theta \in \Theta\}
$$

where $\Theta$ is a smooth $m-1$-dimensional submanifold of the standard $m$-sphere. We will also use the notation $C(\sigma)(\sigma>0)$, for the truncated cone $C \cap B^{m+1}(\sigma)$ where $B^{m+1}(\sigma)$ is the ball of radius $\sigma$ centered at the origin in $\mathbf{R}^{m+1}$. We will frequently use the notions of stability, strict stability, and area minimizing, for hypersurfaces in Riemannian manifolds, as well as strict minimizing for cones in $\mathbf{R}^{m+1}$ (see section 2 of $[12$ and also [8]). In the first lemma, we assume that $M$ is as above, and that near $\operatorname{sing}(M) M$ looks like a union of products of regular cones with complementary dimension manifolds. New metrics are then constructed so that $M$ becomes homologically minimizing in a neighborhood.

Lemma 1. Let $M$ and $\left(N, g_{0}\right)$ be as above, and suppose that there exists $\sigma>0$ such that $\mathcal{N}(\sigma)$ is the disjoint union $\mathcal{N}(\sigma)=\bigcup_{i=1}^{k} \mathcal{N}_{i}(\sigma)$, and assume that there are isometries

$$
\Phi_{i}: \mathcal{N}_{i}(\sigma) \rightarrow B^{n_{i}+1}(\sigma) \times \Sigma_{i},
$$

where $\left(\Sigma_{i}, h_{i}\right)$ is a compact Riemannian manifold of dimension $k_{i}, n_{i}+k_{i}=n$, $n_{i} \geq 7, k_{i} \geq 0$, and and the image of $\Phi_{i}$ is endowed with the product metric. Furthermore, assume that

$$
\Phi_{i}\left(M \cap \mathcal{N}_{i}(\sigma)\right)=C_{i}(\sigma) \times \Sigma_{i}
$$

where $C_{i}$ is any strictly stable, strictly minimizing, regular hypercone in $\mathbf{R}^{n_{i}+1}$.

Then, there exists a metric $g$ on $N$, with $g \equiv g_{0}$ on $\mathcal{N}\left(\sigma_{1}\right)$ for some $\sigma_{1}<\sigma$, and a $\delta>0$, such that $M$ is the unique, homologically area minimizing current in $\mathcal{U}(\delta)$ relative to the metric $g$.

By homologically minimizing area in $\mathcal{U}(\delta)$, we mean that $M$, as considered as a multiplicity one, rectifiable current in $\mathcal{U}(\delta)$ minimizes mass among all rectifiable currents in $\mathcal{U}(\delta)$ homologous to $M$. Note of course, that the hypotheses on $M$ imply that $M$ is already area minimizing in a neighborhood of $\operatorname{sing}(M)$, with $\operatorname{sing}(M)=$ $\bigcup_{i=1}^{k} \Phi_{i}^{-1}\left(0 \times \Sigma_{i}\right)$; the lemma allows us to change the metric away from the singular set so that $M$ becomes locally homologically minimizing. Most of the technical difficulties in the construction have already been carried out in [12], and the needed modifications will be pointed out here. The proof follows from three propositions.

Proposition 1. There is a metric $g_{1}$ on $N$, with that $g_{1} \equiv g_{0}$ on $\mathcal{N}(\sigma / 2)$, such that $M$ is stationary in $\left(N, g_{1}\right)$.

Proof. We will conformally change $g_{0}$ in a neighborhood of $M$ away from $\operatorname{sing}(M)$. That is, $g_{1}$ will have the form $g_{1}=u_{1} g_{0}$, where $u_{1}$ is a smooth, positive function 
on $N$, with $u_{1} \equiv 1$ outside a neighborhood of $M / \mathcal{N}(\sigma)$. Let $H_{0}$, and $H_{1}$ denote the mean curvature functions of $M$ relative to the metrics $g_{0}$ and $g_{1}$ respectively, and let $\nu$ denote the outward unit normal to $M$ (giving the positive orientation). Then, as computed in (2.9) of [12], we have

$$
H_{1}=u_{1}^{-1 / 2}\left(H_{0}-\frac{n}{2 u_{1}} \frac{\partial u_{1}}{\partial \nu}\right) .
$$

Thus $M$ will be stationary relative to $g_{1}\left(H_{1} \equiv 0\right)$, provided that

$$
\frac{1}{u_{1}} \frac{\partial u_{1}}{\partial \nu}=\frac{2 H_{0}}{n} \quad \text { on } M .
$$

There are many functions that satisfy (1.1) and the requirements of the proposition. For example, let $(x, t)$ denote Fermi coordinates for $N$ about $M / \mathcal{N}(\sigma / 4)$. That is $x \in M / \mathcal{N}(\sigma / 4)$ and $t \in(-\epsilon, \epsilon)$ for some $\epsilon>0$, and $t \rightarrow(x, t)$ is a unit speed geodesic perpendicular to $M$ at each $x \in M$. Thus we can assume that $\frac{\partial}{\partial t}=\frac{\partial}{\partial \nu}$. Let $u_{1}$ be a smooth positive function on $N$ such that

$$
u_{1}(x, t)=\left\{\begin{array}{l}
\exp \left(2 / n \int_{0}^{t} H_{0}(x, s) d s\right) \\
1 \quad \text { for }|t| \geq 3 \epsilon / 4 .
\end{array} \quad \text { for } t \in(-\epsilon / 2, \epsilon / 2),\right.
$$

Since $H_{0} \equiv 0$ on $M \cap \mathcal{N}(\sigma), u_{1}$ is globally defined and clearly satisfies (1.1), and also $u_{1} \equiv 1$ on $\mathcal{N}(\sigma / 2)$ if $\epsilon$ is taken sufficiently small, depending on $M$ and $\sigma$.

Proposition 2. There exists a metric $g_{2}=u_{2} g_{1}$, where $u_{2}$ is a smooth positive function on $N$, with $u_{2} \equiv 1$ on $\mathcal{N}\left(\sigma_{1}\right)$ for some $\sigma_{1}<\sigma / 2$, such that $M$ is strictly stable in $\left(N, g_{2}\right)$.

Proof. With minor changes in notation, this is a special case of Lemma 1 in section 2 of [12. Note that $M$ satisfies the hypotheses of Lemma 1 of [12, being minimal in $\left(N, g_{1}\right)$ (from Proposition 1), and also $M \cap \mathcal{N}(\sigma)$ is strictly stable in the sense (2.5) of 12 by the hypotheses on the structure of $M$ near $\operatorname{sing}(M)$. In Lemma 1 of [12], $g_{2}$ is constructed by creating negative Ricci curvature in $N$, in the direction normal to $M$, away from $\operatorname{sing}(M)$, to make the Jacobi operator strictly positive.

The next proposition is analogous to Lemma 4, in section 3 of [12, and states that if $M$ is stricly stable with a singular structure as above, then it is actually locally homologically area minimizing.

Proposition 3. There exists a $\delta>0$, such that $M$ is homologically area minimizing in $\mathcal{U}(\delta)$ relative to the metric $g_{2}$.

Proof. The proof is similar to the proof of Lemma 4 of [12], with some modifications. Since $M$ is orientable, $M$ divides $\mathcal{U}(\delta)$ into two disjoint open components, $\mathcal{U}^{+}(\delta)$ and $\mathcal{U}^{-}(\delta)$ for $\delta$ sufficiently small. Let $(x, t)$ denote Fermi coordinates for $N$ about $M$ as in the proof of Proposition 1, valid for $|t|<\delta$, and for $x \in M / \mathcal{N}\left(\sigma_{1} / 8\right)$, again for $\delta$ sufficiently small, depending on $M,\left(N, g_{2}\right)$ and $\sigma_{1}$. Here also, $t$ will be oriented so that $(x, t) \in \mathcal{U}^{+}(\delta)$ for $t>0$. We will construct barrier hypersurfaces on both sides of $M$. Let $\rho=\rho(x)=\operatorname{dist}(x, \operatorname{sing}(M))$ for $x \in M$. For $|t|<\delta$, let

$$
\Gamma_{t}=\left\{(x, t): x \in M, \rho(x) \geq \sigma_{1} / 8\right\},
$$


That is, $\Gamma_{t}$ is the graph of the constant function $t$ over $M$. Letting $h_{t}^{\Gamma}$ denote the mean curvature function of $\Gamma_{t}$, we have as in (3.1) of [12],

$$
\left|h_{t}^{\Gamma}\right| \leq c|t|
$$

for some constant $c$ (depending on $\sigma$ ), as $M$ is minimal. To construct the barriers near $\operatorname{sing}(M)$, define for each $i=1, \ldots, k$, and $|t|$ sufficiently small (depending on $\left.\sigma_{1}\right)$

$$
\Lambda_{t}^{i}=\left\{(x, t): \rho(x, 0)=\sigma_{1} / 4\right\} .
$$

Let $R_{t}^{i}$ be the area minimizing $n$-current in $\mathcal{N}_{i}\left(\sigma_{1} / 4\right)$ with $\partial R_{t}^{i}=\Lambda_{t}^{i}, i=1, \ldots, k$. By abuse of notation, we will also denote by $R_{t}^{i}$ the support of $R_{t}^{i}$. Note that by our hypotheses on $(N, g)$ and $M$, that $\Phi_{i}\left(R_{t}^{i}\right)$ is area minimizing in $B^{n_{i}+1}\left(\sigma_{1} / 2\right) \times \Sigma_{i}$ with the product metric, with boundary the product

$$
\partial \Phi_{i}\left(R_{t}^{i}\right)=\operatorname{graph}_{\partial C_{i}\left(\sigma_{1} / 4\right)}\{t\} \times \Sigma_{i} .
$$

Therefore $\Phi_{i}\left(R_{t}^{i}\right)$ is a product

$$
\Phi_{i}\left(R_{t}^{i}\right)=S_{t}^{i} \times \Sigma_{i},
$$

where $S_{t}^{i} \subset B^{n_{i}+1}\left(\sigma_{1} / 2\right)$ as an area minimizing $n_{i}$-current whose boundary is $\operatorname{graph}\{t\}$ over $\partial C_{i}(\sigma / 4)$. By [8], $\operatorname{spt} S_{t}^{i}$ is a smooth hypersurface lying on one side of $C_{i}$. Furthermore, there is a $r_{t}>0$ with $r_{t} \rightarrow 0$ as $|t| \rightarrow 0$, such that for $x \in B^{n_{i}+1}\left(\sigma_{1} / 2\right)$ with $r_{t} \leq|x| \leq \sigma_{1} / 4, S_{t}^{i}$ is the graph over $C_{i}$ of a smooth function $w_{i}$. Thus, after composition with $\Phi^{-1}$, we see that $R_{t}^{i}$ is the graph over $M$ for $r_{t} \leq \rho \leq \sigma_{1} / 4$, of a smooth function $f_{i}$ (which is independent of the $\Sigma_{i}$ variable). Denote by $R_{t}$ the union $\bigcup_{i} R_{t}^{i}$. Of course the mean curvature of $R_{t}$ is identically zero, and from above, $R_{t}$ is the graph over $M$ for $r_{t} \leq \rho \leq \sigma_{1} / 2$ of some smooth function $\psi_{t}$. As in [12, we now glue together the $R_{t}$ to the $\Gamma_{t}$ along $\sigma_{1} / 4 \leq \rho \leq \sigma_{1}$. Note that by the Harnack inequality (since $\psi_{t}>0$ for $t>0$ ) we have

$$
c^{-1}|t| \leq\left|\psi_{t}\right| \leq c|t|, \quad \text { for } \sigma_{1} / 8 \leq \rho \leq \sigma_{1} / 4,
$$

for some constant $c$. By standard elliptic estimates [7, we then have

$$
\max _{\sigma_{1} / 7 \leq \rho \leq \sigma_{1} / 5}\left|\nabla^{j} \psi_{t}\right| \leq c|t|, \quad k=1,2 .
$$

Let $\zeta$ be a smooth non-negative function on $M$ such that

$$
\zeta(x)= \begin{cases}0 & \text { for } \rho \geq \sigma_{1} / 5 \\ 1 & \text { for } \rho \leq \sigma_{1} / 7\end{cases}
$$

and let $u_{t}$ be the smooth function defined on $M /\left\{\rho<\sigma_{1} / 8\right\}$

$$
u_{t}=\zeta \psi_{t}+(1-\zeta) t \text {. }
$$

It follows from the above estimates on $\psi_{t}$ and the definition of $u_{t}$ that

$$
\begin{gathered}
c^{-1}|t| \leq\left|u_{t}\right| \leq c|t| \quad \text { for } \rho \geq \sigma_{1} / 8, \\
\max _{\sigma_{1} / 7 \leq \rho \leq \sigma_{1} / 5}\left|\nabla^{k} u_{t}\right| \leq c|t|, \quad k=1,2 .
\end{gathered}
$$

Note that the graph of $u_{t}$ (that is, in Fermi coordinates, the set $\left\{\left(x, u_{t}(x)\right): x \in\right.$ $\left.\left.M, \rho(x) \geq \sigma_{1} / 8\right\}\right)$ contains the portion of $\Gamma_{t}$ corresponding to $\rho(x) \geq \sigma_{1} / 5$, and the graph of $\psi_{t}$ for $\sigma_{1} / 8 \leq \rho(x) \leq \sigma_{1} / 7$. Let $\Omega_{t}$ be the smooth hypersurface obtained taking the union of the graph of $u_{t}$ with the part of $R_{t}$ corresponding to $\rho \leq \sigma_{1} / 8$. From here the proof proceeds as in the proof of Lemma 4 of [12], and 
thus a concise sketch will be given here. By the comments above on the structure of $R_{t}$, it follows that $\Omega_{t}$ converges to $M$ in Hausdorff distance as $t \rightarrow 0$. For $t>0$, let $V_{t}$ be the open component of $N /\left(\Omega_{t} \cup \Omega_{-t}\right)$, that contains $M$. In proving the proposition, we may replace $\mathcal{U}(\delta)$ by $V_{t}$, since given $\delta>0$ there is a $t>0$ such that $V_{t} \subset \mathcal{U}(\delta)$, and conversely, given $t>0$, there is a $\delta>0$ such that $\mathcal{U}(\delta) \subset V_{t}$. Proving by contradiction, suppose that the proposition was false. Then there is a sequence $t_{j} \rightarrow 0$, and $n$-currents $T_{j}$ in $\bar{V}_{t_{j}}$ such that $\mathbf{M}\left(T_{j}\right)<\mathbf{M}(M)$, with $T_{j}$ homologous to $M$. Here $\mathbf{M}$ denotes the mass, and $M$ is considered as a multiplicity one current. We may also assume by the compactness theorems of integral currents [6], that $T_{j}$ minimizes mass among all $n$ currents in $\bar{V}_{t_{j}}$ homologous to $M$. Since $\mathbf{M}\left(T_{j}\right)$ is bounded in $j$, there is a subsequence, still denoted by $T_{j}$ and an $n$ current $T$, homologous to $M$, such that $T_{j} \rightarrow T$ in the sense of currents. Clearly, $\operatorname{spt}(T) \subset \bigcap_{j} \bar{V}_{t_{j}}=M$, and so by the constancy theorem, we must have $T=M$. Note that $T_{j}$ minimizes mass relative to the obstacle $\partial V_{t_{j}}=\Omega_{t_{j}} \cup \Omega_{-t_{j}}$, and so by [13, $\operatorname{spt}\left(T_{j}\right)$ is actually a $C^{1,1 / 2}$ manifold outside a singular set of dimension less than $n-2$, and the mean curvature of $T_{j}$ is bounded by the mean curvature of $\Omega_{t_{j}} \cup \Omega_{-t_{j}}$ which is bounded by $c\left|t_{j}\right| \rightarrow 0$, for some constant $c$ independent of $j$. It then follows from Allard's regularity theorem ([1] or 24.2 of [9]) that there exists $\delta_{j}>0$, with $\delta_{j} \rightarrow 0$, and $C^{1,1 / 2}$ functions $v_{j}$ on $M / \mathcal{N}\left(\delta_{j}\right)$ such that for all $j$ sufficiently large, $\operatorname{spt}\left(T_{j}\right) / \mathcal{N}\left(\delta_{j}\right)=\operatorname{graph}\left(v_{j}\right)$. The idea of the proof is to show that $\delta_{j}$ can be taken sufficiently small, so that $v_{j}$ can be extended to all of $M$ in such a way that the graph of $v_{j}$ has smaller area than $M$, for large $j$ (using of course $\mathbf{M}\left(T_{j}\right)<\mathbf{M}(M)$ ). This will then contradict strict stability of $M$ (Proposition 2) which says more or less that $M$ has smaller area than nearby graphs. Define $s_{j}$ by

$$
s_{j}=\max _{\rho \geq \sigma_{1} / 8}\left|v_{j}\right| \leq c t_{j}
$$

for some constant $c$ independent of $j$. Let $i \in\{1, \ldots, k\}$ be fixed, and denote by $\hat{T}_{j}$ the restriction of $T_{j}$ to $\mathcal{N}_{i}\left(\sigma_{1}\right)$. Let $W_{j}$ be the current

$$
W_{j}=\Phi_{i_{\sharp}}\left(\hat{T}_{j}\right) .
$$

Thus $\operatorname{spt} W_{j} \subset B^{n_{i}+1}\left(\sigma_{1} / 4\right) \times \Sigma_{i}$, and lies between the barriers $S_{-s_{j}}^{i} \times \Sigma_{i}$ and $S_{s_{j}}^{i} \times \Sigma_{i}$. Since the barriers are area minimizing in $B^{n_{i}+1} \times \Sigma_{i}$, it follows from [13] that $W_{j}$ is also area minimizing. But then by the maximum principle [10], $\operatorname{spt} W_{j}$ lies between $\lambda_{j}^{ \pm} S^{ \pm} \times \Sigma_{i}$, inside $B^{n_{i}+1}\left(\sigma_{1} / 8\right) \times \Sigma_{i}$, where $S^{ \pm}$are the smooth area minimizing hypersurfaces in $\mathbf{R}^{n_{i}+1}$ that lie on either side of $C_{i}$ described in the proposition of $\S 3$ of [12] (see also $\S 2$ of [8]), and $\lambda_{j}^{ \pm}$are appropriate scalings depending on $s_{j}$. The choice of $\lambda_{j}^{ \pm}$is made so that the minimum height of $S^{ \pm}$over $C_{i}$ at $r=\sigma_{1} / 8$ is $s_{j}$. Recall that $S^{ \pm} \cap \mathbf{R}^{n_{i}+1} /\left\{r<R_{0}\right\}$ is the graph over $C_{i} / C_{i}\left(R_{0}\right)$ of a function $u^{ \pm}$, for some $R_{0}>0$. Thus $\lambda_{j}^{ \pm} S^{ \pm} \cap \mathbf{R}^{n_{i}+1} /\left\{r<R_{j}^{ \pm}\right\}$is the graph of some function $u_{j}$, and some $R_{j}^{ \pm} \rightarrow 0$. In fact, using the asymptotic behavior of $u^{ \pm}$ from $\left[8\right.$, it was shown in $\S 3$ of [12], that $\lambda_{j}^{ \pm}$and $R_{j}^{ \pm}$can be chosen so that

$$
c^{-1} s_{j}^{\frac{1}{1-\gamma}} \leq \lambda_{j}^{ \pm} \leq c s_{j}^{\frac{1}{1-\gamma}}
$$

and

$$
R_{j}=R_{j}^{ \pm}=b s_{j}^{\frac{1}{1-\gamma}}
$$


for some constants $b$ and $c$, and where

$$
\gamma=\frac{2-n_{i}}{2}+\sqrt{\frac{\left(n_{i}-2\right)^{2}}{4}+\mu_{1}}
$$

with $\mu_{1}$ a constant depending on $C_{i}$, with the quantity inside the radical positive (due to strict stability of $C_{i}$ ). Now, let $\epsilon>0, \epsilon<<1$, to be fixed later, and let $\sigma_{j}=R_{j}^{1-\epsilon}$. Then we have (see (3.13) [12]),

$$
\left|u_{j}(y)\right| \leq c \sigma_{j}^{\frac{1-\epsilon \gamma}{1-\epsilon}} \leq c \sigma_{j}^{1+\beta},
$$

for some constant $c$, some $\beta>0$ depending on $\epsilon$, and for $\sigma_{j} \leq|y| \leq \sigma_{1} / 4, y \in C_{i}$. We therefore have

$$
\operatorname{dist}\left(\operatorname{spt} W_{j} \cap\left(B^{n_{i}+1}\left(\sigma_{1} / 8\right) / B^{n_{i}+1}\left(\sigma_{j}\right) \times \Sigma_{i}\right), C_{i}\left(\sigma_{1} / 8\right) / C_{i}\left(\sigma_{j}\right) \times \Sigma_{i}\right) \leq c \sigma_{j}^{1+\beta},
$$

where dist is the Hausdorff distance. It now follows that $\operatorname{spt} W_{j} \cap\left(B\left(\sigma_{1} / 8\right) / B\left(\sigma_{j}\right) \times\right.$ $\left.\Sigma_{i}\right)$ is a graph over $C_{i}\left(\sigma_{1} / 8\right) / C_{i}\left(\sigma_{j}\right) \times \Sigma_{i}$ for large $j$. Arguing by contradiction, suppose this was not the case. Then there are points $p_{j}=\left(q_{j}, m_{j}\right) \in C_{i} \times \Sigma_{i}$, $j \rightarrow \infty$, with $\sigma_{j} \leq\left|q_{j}\right| \leq \sigma_{1} / 8$, such that $\operatorname{spt} W_{j}$ is not a graph over the point $p_{j}$. After taking a subsequence, we may assume that

$$
\left|q_{j}\right|^{-1} q_{j} \rightarrow q \in C_{i} \quad \text { and } \quad m_{j} \rightarrow m \in \Sigma_{i}
$$

and we also may assume that $\left|q_{j}\right| \rightarrow 0$ as $j \rightarrow \infty$; otherwise the desired result follows immediately from the above inequality and Allards regularity theorem (see [1] or chapter 5 of 9]). But then, if we let $A$ be a normal coordinate neighborhood of $m$ in $\Sigma_{i}$, the above inequality implies that

$$
\left|q_{j}\right|^{-1}\left(\operatorname{spt} W_{j} \cap\left(B\left(\sigma_{1} / 8\right) / B\left(\left|q_{j}\right| / 2\right) \times A\right)\right) \rightarrow C_{i} / C_{i}(1 / 2) \times \mathbf{R}^{k_{i}},
$$

as $j \rightarrow \infty$. But then, as above, Allard's theorem implies that for $j$ sufficiently large, $\operatorname{spt} W_{j}$ is graphical over $C_{i} \times \Sigma_{i}$ near $p_{j}$. Of course $\sigma_{j}$ and $\gamma$ depend on $i$; denote

$$
\sigma_{j}=\sigma_{j}(i), \quad \gamma=\gamma(i), \quad \text { for } i=1, \ldots, k, j \rightarrow \infty .
$$

Thus, after composition with $\Phi_{i}, v_{j}$ is defined on $M \cap \mathcal{N}_{i}\left(\sigma_{1}\right)$ for $\rho \geq \sigma_{j}(i)$, and furthermore we have the estimate

$$
\left|v_{j}(x)\right| \sigma_{j}(i)^{-1-\beta}+\left|\nabla v_{j}(x)\right| \sigma_{j}(i)^{-\beta} \leq c \quad \text { for } x \in \mathcal{N}\left(\sigma_{1}\right), \rho \geq \sigma_{j},
$$

for a constant $c$ independent of $j$. The first term on the left is bounded since $\left|v_{j}\right|$ is bounded by $\left|u_{j}^{ \pm}\right|$over this domain, while the bound on the second term follows from the bound on the first, and on standard elliptic estimates [7]. Now we extend $v_{j}$ to a function $\tilde{v}_{j}$ defined on all of $M$ (see 3.15) of [12] such that

$$
\begin{gathered}
\tilde{v}_{j}(x)=v_{j}(x) \quad \text { for } x \in M / \bigcup_{i=1}^{k} \mathcal{N}\left(2 \sigma_{j}(i)\right), \\
\tilde{v}_{j}(x)=0 \quad \text { for } x \in \bigcup_{i=1}^{k} \mathcal{N}\left(\sigma_{j}(i)\right), \\
\left|\tilde{v}_{j}(x)\right| \sigma_{j}(i)^{-1-\beta}+\left|\nabla \tilde{v}_{j}(x)\right| \sigma_{j}(i)^{-\beta} \leq c \quad \text { for } x \in \bigcup_{i=1}^{k} \mathcal{N}\left(2 \sigma_{j}(i)\right) / \mathcal{N}\left(\sigma_{j}(i)\right),
\end{gathered}
$$

for some constant $c$. Let $\tilde{T}_{j}$ be the multiplicity one current corresponding to the graph of $\tilde{v}_{j}$, oriented so that it is homologous to $M$. Now, $\operatorname{spt} \tilde{T}_{j}=\operatorname{spt} T_{j}$ outside of 
$\bigcup_{i} \mathcal{N}_{i}\left(2 \sigma_{j}(i)\right)$, and the masses of each, inside $\mathcal{N}_{i}\left(2 \sigma_{j}(i)\right)$ are bounded by a constant times $\sigma_{j}(i)^{n_{i}}$, and therefore, the mass of $\tilde{T}_{j}$ inside $\mathcal{N}_{i}\left(2 \sigma_{j}(i)\right)$, is bounded by

$$
c \sum_{i=1}^{k} \sigma_{j}(i)^{n_{i}} \leq c \sum_{i=1}^{k} s_{j}^{\frac{1-\epsilon}{1-\gamma_{i}} n_{i}},
$$

for some constant $c$. However, by the definition of $\gamma_{i}$ one can fix $\epsilon>0$ such that

$$
\frac{1-\epsilon}{1-\gamma_{i}} n_{i}>2, \quad \text { for } i=1, \ldots, k .
$$

It then follows that there is a $\beta_{1}>0$, such that

$$
\mathbf{M}\left(\tilde{T}_{j}\right)-\mathbf{M}\left(T_{j}\right) \leq c s_{j}^{2+\beta_{1}} .
$$

The rest of the argument now proceeds exactly as in $\S 3$ of 12 . Letting $A(v)$ denote the area of the graph of a $C^{1}$ function $v$ on $M$, with sufficiently small $C^{1}$ norm, supported away from $\operatorname{sing}(M)$, we have by Taylors theorem, and the first and second variation formulas (together with $M$ stationary),

$$
A\left(\tilde{v}_{j}\right)=A(0)+\int_{M}\left|\nabla \tilde{v}_{j}\right|^{2}-\left(|B|^{2}+R\right) \tilde{v}_{j}^{2} d v+E\left(\tilde{v}_{j}\right),
$$

where $|B|^{2}$ is the second fundamental form of $M$ squared, $R$ is the Ricci curvature of $N$ in the normal direction of $M, d v$ is the volume form of $M$, and $E$ is the third order remainder. But strict stability of $M$ (see $\S 2$ of [12]) implies that

$$
\int_{M}\left|\nabla \tilde{v}_{j}\right|^{2}-\left(|B|^{2}+R\right) \tilde{v}_{j}^{2} d v \geq \mu \int_{M} \tilde{v}_{j}^{2} \rho^{-2} d v
$$

for a positive constant $\mu$. A computation of $E\left(\tilde{v}_{j}\right)((3.20)-(3.29)$ of [12]), together with Allards regularity theorem, standard Schauder estimates, and the above estimates on $v_{j}$, reveals that (see (3.19) [12])

$$
E\left(\tilde{v}_{j}\right) \leq \mu / 10 \int_{M} \tilde{v}_{j}^{2} \rho^{-2} d v,
$$

for sufficiently large $j$. Therefore we have,

$$
\mathbf{M}\left(\tilde{T}_{j}\right) \geq \mathbf{M}(M)+\mu / 4 \int_{M} \tilde{v}_{j}^{2} \rho^{-2} d v
$$

again for $j \rightarrow \infty$. However, $\tilde{v}_{j}$ satisfies the estimate $((3.18)$ of [12])

$$
\int_{M} \tilde{v}_{j}^{2} \rho^{-2} d v \geq c s_{j}^{2}
$$

which follows from elliptic estimates for solutions of divergence form, quasilinear elliptic equations [7], after one notes that (weakly)

$$
\mathcal{H}\left(v_{j}\right)=h_{j} \quad \text { on } M / \mathcal{N}\left(\sigma_{1} / 8\right)
$$

where $\mathcal{H}$ is the mean curvature operator on $M$, and that the mean curvature $h_{j}$ of $v_{j}$ is bounded by a constant times $s_{j}$. Thus (1.3) becomes

$$
\mathbf{M}\left(\tilde{T}_{j}\right) \geq \mathbf{M}(M)+c s_{j}^{2},
$$

for a positive constant $c$, and $j$ large. Combining this with (1.2), and taking $j$ large, this implies that

$$
\mathbf{M}(M)<\mathbf{M}\left(T_{j}\right)
$$


contradicting our assumption, which finishes the proof of Proposition 3, and thus of Lemma 1.

Lemma 2. Let $\left(N, g_{0}\right)$ be smooth, compact, $n+1$-dimensional Riemannian manifold, and suppose that $M$ is a multiplicity one integral $n$-current which is uniquely homologically area minimizing in some neighborhood $\mathcal{U}$ of sptM, and that $M$ is homologically nontrivial in $N$. Then there is a smooth Riemannian metric $g$ on $N$, such that $g \equiv g_{0}$ on some smaller neighborhood $\mathcal{V} \subset \mathcal{U}$, of sptM, such that relative to $g, M$ is homologically area minimizing in $N$.

Such metrics were constructed in $\S 4$ of $[12$. There, $M$ was a specific hypersurface with two isolated singularities that had the hypotheses of the lemma, however the singular structure of $M$ was never used in the proof, only the fact that $M$ was uniquely homologically minimizing in a neighborhood $\mathcal{U}$. Then a smaller neighborhood $\mathcal{V}^{\prime}$ of $M$ was chosen with two smooth boundary components. It was then shown that if $W$ was any smooth compact, connected manifold with $\partial W$ diffeomorphic to $\partial \mathcal{V}^{\prime}$, then $W$ could be smoothly connected to $\mathcal{V}^{\prime}$ along their boundaries, and a metric could be put on the resulting manifold (identical to the original metric on a smaller neighborhood $\mathcal{V}$ of $M$ ), in such a way that $M$ was globally homologically area minimizing. Thus the same construction could be applied here where $W$ is simply the complement in $N$ of such a neighborhood $\mathcal{V}^{\prime}$ of $M$ with $\mathcal{V}^{\prime} \subset \mathcal{U}$.

\section{Constructing global homological minimizers}

We first state and prove Theorem 1, which implies Theorem A of the introduction. For convenience, we will denote by $\mathcal{F}(n+1), n \geq 7$, the following class of smooth manifolds. A manifold $\Sigma$ will be in $\mathcal{F}(n+1)$ if and only if $\Sigma$ is smooth, connected, compact, orientable, has dimension less than or equal to $n-7$ and satisfies the the topological condition that there exists a smooth embed-

$\operatorname{ding} \Phi: \bar{B}^{m+1} \times \Sigma \rightarrow \mathbf{R}^{n+1}$, where $m+1+\operatorname{dim} \Sigma=n+1$, and where $\bar{B}^{m+1}$ is the closure of the open unit ball in $\mathbf{R}^{m+1}$.

Remark. One can easily check that if $\Sigma$ is any standard sphere or torus of dimension less than or equal to $n-7$, then $\Sigma \in \mathcal{F}(n+1)$, and thus Theorem 1 implies Theorem A. In fact, it is not hard to see, that if there exists an embedding of $\Sigma$ into $\mathbf{R}^{n+1}$ with trivial normal bundle, then $\Sigma \in \mathcal{F}(n+1)$. In particular, if $\operatorname{dim} \Sigma=k \leq n-7$, and there is an embedding of $\Sigma$ into $\mathbf{R}^{k+1}$, then $\Sigma \in \mathcal{F}(n+1)$ (assuming of course that $\Sigma$ is smooth, connected and compact).

Theorem 1 allows us to construct homologically area minimizing hypersurfaces, with singular set any finite union of submanifolds belonging to $\mathcal{F}(n+1)$.

Theorem 1. Let $N$ be a smooth, compact manifold of dimension $n+1 \geq 8$, and let $S$ be a smooth, embedded, connected, orientable submanifold of $N$ of dimension $n$, such that $S$ is homologically non-trivial. Let $\left(\Sigma_{1}, h_{1}\right), \ldots,\left(\Sigma_{L}, h_{L}\right), L \geq 1$, be any collection of Riemannian manifolds such that $\Sigma_{i} \in \mathcal{F}(n+1)$, and define $k_{i}=\operatorname{dim} \Sigma_{i}$, $i=1, \ldots, L$. Let $C_{1}, \ldots, C_{L}$ be any collection of strictly stable, strictly minimizing regular hypercones, $C_{i} \subset \mathbf{R}^{n_{i}+1}$, with $n_{i}=n-k_{i}$. Then there is a metric $g$ on $N$, such that the area minimizing current homologous to $S$, is a multiplicity one, 
connected hypersurface $M$, with

$$
\operatorname{sing}(M)=\bigcup_{i=1}^{L} \Lambda_{i},
$$

where $\Lambda_{i}$ is an embedded copy of $\Sigma_{i}, i=1, \ldots, L$. Furthermore, near $\Lambda_{i}, N$ is isometric to $B^{n_{i}+1}(\sigma) \times \Sigma_{i}$ with the product metric $\delta \times h_{i}$ ( $\delta$ being the Euclidean metric) with $M$ isometric to $C_{i}(\sigma) \times \Sigma_{i}$, for some $\sigma$ sufficiently small.

Proof. The following proposition is useful in the construction of $M$. It says essentially, that we can "cap off" a truncated cone, and follows from some elementary facts from topology.

Proposition. Let $C$ be a regular minimal hypercone in $\mathbf{R}^{m+1}, m \geq 2$. Then there exists a compact hypersurface, without boundary, $\hat{C} \subset \mathbf{R}^{m+1}$, which is smoothly embedded except at the origin, and a $\sigma>0$, such that $\hat{C} \cap B^{m+1}(\sigma)=C \cap B^{m+1}(\sigma)$. Furthermore, $\hat{C}$ is contained in the unit ball.

Proof of Proposition. Consider the truncated cone $C(1)=B^{m+1}(1) \cap C$. The boundary, $\partial C(1)$ is a smoothly embedded $m$-1-dimensional submanifold of $\mathbf{R}^{m+1}$, and therefore (from basic results of topology) it bounds a smooth, compact $n$ dimensional manifold $X$. Then $Y=X \cup C(1)$ is a piecewise, $C^{1}$, compact embedded hypersurface, away from $\{0\}$. Now, outside of a sufficiently small ball $B^{m+1}(\epsilon)$, approximate $Y$ by a smooth embedding, whose image we denote by $\hat{Y}$, such that $\hat{Y} \cap B^{m+1}(2 \epsilon)=Y \cap B^{m+1}(2 \epsilon)$. Finally, if we set $\hat{C}$ to be a sufficiently small scaling of $\hat{Y}$, then $\hat{C}$ satisfies the requirements of the proposition.

Now, since $S$ is orientable, there is a neighborhood $\mathcal{E}$ of $S$, such that $S$ divides $\mathcal{E}$ into two components, $\mathcal{E}^{+}$and $\mathcal{E}^{-}$. Let $p_{1}, \ldots, p_{L}$ be in $S$, and let $\mathcal{B}_{1}, \ldots, \mathcal{B}_{L}$ be pairwise disjoint open sets of $N$ diffeomorphic to $n+1$-balls, such that for each $i$, $p_{i} \in \mathcal{B}_{i} \subset \mathcal{E}$, and so that $S$ divides $\mathcal{B}_{i}$ into two open components $\mathcal{B}^{ \pm} \subset \mathcal{E}^{ \pm}$.

For each $i=1, \ldots, L$, we will do the following construction. Let $\hat{C}_{i}$ be a capped off $C_{i}$ in $B^{n_{i}+1}$ (the unit ball in $\mathbf{R}^{n_{i}+1}$ centered at the origin) as in the proposition. It follows from the hypotheses on $\Sigma_{i}$, that there is an embedding (into) $\Phi_{i}: B^{n_{i}+1} \times$ $\Sigma_{i} \rightarrow \mathcal{B}_{i}^{+}$. Denote by $M_{i}=\Phi_{i}\left(\hat{C}_{i} \times \Sigma_{i}\right)$, and let $D_{i}$ be a $n$-disc in $M_{i}$ in the image of $\left(B^{n_{i}+1}(1) / B^{n_{i}+1}(1 / 2)\right) \times \Sigma_{i}$, and let $D_{i}^{\prime}$ be a $n$-disc in $S \cap \mathcal{B}_{i}$. Delete $D_{i}$ and $D_{i}^{\prime}$, and smoothly connect $S$ and $M_{i}$ by a handle (a hypersurface in $N$ diffeomorphic to an $n-1$-sphere times an interval) in $\mathcal{B}_{i}^{+}$, glueing the boundaries of the handle onto $D_{i}$ and $D_{i}^{\prime}$. Let $M$ be the resulting hypersurface in $N$, that is the connected sum of $S$ with $M_{i}, i=1, \ldots, L$. The connected sum operation can be done so that $M$ is embedded away from $\operatorname{sing}(M)$. Note of course that then, $M$ is orientable, and homologous to $S$ (as a current) if given the right orientation. Let $g_{i}$ be the pullback of the product metric on $\Phi_{i}\left(B^{n_{i}+1} \times \Sigma_{i}\right)$. That is, if $\delta_{i}$ denotes the Euclidean metric on $\mathbf{R}^{n_{i}+1}$, then set

$$
g_{i}=\left(\Phi_{i}^{-1}\right)^{*}\left(\delta_{i} \times h_{i}\right)
$$

Using a standard partition of unity argument, we can then put a metric $g_{0}$ on $N$ such that on each $\Phi_{i}\left(B^{n_{i}+1}(1 / 2) \times \Sigma_{i}\right), g_{0}=g_{i}, i=1, \ldots, L$. But then, $\left(N, g_{0}\right)$ and $M$ satisfy the hypotheses of Lemma 1 , and we can thus find a metric $h$ on $N$, with $h \equiv g_{0}$ on a smaller neighborhood of $\operatorname{sing}(M)$, such that $M$ is homologically minimizing in a tubular neighborhood. But then, we can apply Lemma 2 to find a metric $g$ on $N$ which satisfies the requirements of Theorem 1 . 
The next theorem allows us to construct examples of homologically minimizing hypersurfaces, with singular sets of essentially arbitrary topological type (at least in the category of smooth submanifolds of appropriate codimension). However, unlike Theorem 1, we cannot prescribe the homology class or the ambient manifold.

Theorem 2. Let $n \geq 7$, and let $\left(\Sigma_{1}, h_{1}\right), \ldots,\left(\Sigma_{L}, h_{L}\right)$ be smooth, compact, orientable Riemannian manifolds, with $\operatorname{dim} \Sigma_{i}=k_{i} \leq n-7$. Let $X_{1}, \ldots, X_{L}$ be smooth, compact orientable manifolds with $\operatorname{dim} X_{i}=n_{i}+1$ where $n_{i}+k_{i}=n$, and let $C_{1}, \ldots, C_{L}$ be strictly stable, strictly minimizing, regular hypercones with $C_{i} \subset \mathbf{R}^{n_{i}+1}, i=1, \ldots, L$. Then we can construct an $n+1$-manifold $N$ by forming a connected sum $\left(X_{1} \times \Sigma_{1}\right) \# \cdots \#\left(X_{L} \times \Sigma_{L}\right)$, together with a handle attached, and a metric $g$ on $N$, such that $N$ supports a homologically area minimizing current $M$, which is a multiplicity one, connected hypersurface, with $\operatorname{sing}(M)=\bigcup_{i} \Lambda_{i}$ where, for each $i, \Lambda_{i}$ is a diffeomorphic copy of $\Sigma_{i}$ in $N$. Near each $\Lambda_{i}$, M looks (metrically) like the product $C_{i} \times \Sigma_{i}$.

Proof. As in the proof of Theorem 1, let $B^{n_{i}+1}$ denote the unit ball centered at the origin in $\mathbf{R}^{n_{i}+1}$, and let $\hat{C}_{i}$ be a capped off $C_{i}$ in $B^{n_{i}+1}$ (as in the proposition), $i=1, \ldots, L$. For each $i$, let $F_{i}: B^{n_{i}+1} \rightarrow X_{i}$ be a smooth embedding, and let $g_{i}$ be a metric on $X_{i}$ such that

$$
g_{i} \equiv\left(F_{i}^{-1}\right)^{*}\left(\delta_{i}\right) \quad \text { on } F_{i}\left(B^{n_{i}+1}(1 / 2)\right)
$$

and equip $X_{i} \times \Sigma_{i}$ with the product metric $g_{i} \times h_{i}$. Let $D_{i}$ be an $n+1$-disk in $X_{i} \times \Sigma_{i}$ contained in the complement of $F_{i}\left(B^{n_{i}+1}(1 / 2)\right) \times \Sigma_{i}, i=1, \ldots, L$. Now form the connected sum

$$
N^{\prime}=\underset{i=1}{\#}\left(X_{i} \times \Sigma_{i}\right)
$$

by deleting each $D_{i}$ from $X_{i} \times \Sigma_{i}$, and smoothly glueing $\partial D_{i}$ to $\partial D_{i+1}$ (preserving orientation), $i \leq L-1$. Put a metric $g^{\prime}$ on $N^{\prime}$ which coincides with $g_{i} \times h_{i}$ on each $\left(X_{i} \times \Sigma_{i}\right) / D_{i}$. Note that each $F_{i}\left(\hat{C}_{i}\right) \times \Sigma_{i}$ sits in $N^{\prime}$, and form a pairwise disjoint collection of hypersurfaces. Now, for $i=1, \ldots, L-1$, smoothly connect $F_{i}\left(\hat{C}_{i}\right) \times \Sigma_{i}$ to $F_{i+1}\left(\hat{C}_{i+1}\right) \times \Sigma_{i+1}$ by a handle in $N^{\prime}$ (as in the proof of Theorem 1 ), attaching them at the boundaries of $n$-disks, well away from the singular set. Let $M$ denote the resulting hypersurface. Of course, $M$ is homologically trivial in $N^{\prime}$, however, it is easy to see that it divides $N^{\prime}$ into two open components $N_{+}^{\prime}$ and $N_{-}^{\prime}$. Form a smooth, oriented $n+1$-manifold, $N$ by attaching a handle from $N_{+}^{\prime}$ to $N_{-}^{\prime}$. That is, delete $n+1$-disks from $N_{+}^{\prime}$ and $N_{-}^{\prime}$ and let $N$ be the union of these, together with $Y=n-$ sphere $\times(0,1)$, where the boundary components of $Y$ are identified with the boundaries of the deleted disks by suitable diffeomorphisms. Finally, put a metric $G$ on $N$ which coincides with $g^{\prime}$ on $N^{\prime}$. Now $M$ is homologous to a nontrivial $n-$ sphere $\times\{$ point $\} \subset Y \subset N$. Also, $M$ satisfies the hypotheses of Lemma 1 , so we can find a metric $h$ on $N$, which is identical to $G$ in a neighborhood of $\operatorname{sing}(M)$, and so that $M$ is homologically minimizing in a tubular neighborhood. Then, apply Lemma 2, to get a metric $g$ on $N$, which satisfies the requirements of Theorem 2.

\section{REFERENCES}

1. W.K. Allard, On the first variation of a varifold, Ann. Math. 95 (1972), 417-491. [MR 46:6136

2. E. Bombieri, E. De Giorgi, E. Giusti, Minimal cones and the Bernstein problem, Invent. Math. 7 (1969), 243-268. MR 40:3445 
3. L. Caffarelli, R. Hardt, L. Simon, Minimal surfaces with isolated singularities, Manuscripta Math. 48 (1984), 1-18. MR 86h:53007

4. E. De Giorgi, Frontiere orientate di misura minima, Sem. Mat. Scuola Norm. Sup. Pisa (1961), 1-56. MR 31:3897

5. H. Federer, The singular sets of area minimizing rectifiable currents with codimension one and area minimizing flat chains modulo two with arbitrary codimension, Bull. Amer. Math. Soc. 76 (1970), 767-771. MR 41:5601

6. H. Federer, W. Fleming, Normal and integral currents, Ann. Math. 72 (1960), 458-520. MR 23:A588

7. D. Gilbarg, N. Trudinger, Elliptic Partial Differential Equations of Second Order (2nd ed.), Springer-Verlag, 1983. MR 86c:35035

8. R. Hardt, L. Simon, Area minimizing hypersurfaces with isolated singularities, J. Reine Angew. Math. 362 (1985), 102-129. MR 87f:49052

9. L. Simon, Lectures on Geometric Measure Theory, Proceedings for the Centre for Mathematical Analysis, Australian National University, Canberra, 1983. MR 87a:49001

10. L. Simon, A strict maximum principle for area minimizing hypersurfaces, J. Differential Geom. 26 (1987), 327-335. MR 88h:49069

11. J. Simons, Minimal varieties in Riemannian manifolds, Ann. Math. 88 (1968), 62-105. MR 38:1617

12. N. Smale, Singular homologically area minimizing surfaces of codimension one in Riemannian manifolds, Invent. Math. 135 (1999), 145-183. CMP 99:06

13. B. White, Existence of smooth embedded surfaces of prescribed genus that minimize parametric even elliptic functionals on 3-manifolds, J. Differential Geom. 33 (1991), 413-443. MR 92e:58048

Department of Mathematics, University of Utah, Salt Lake City, Utah 84112 